\title{
Provision of a chlamydial culture service to a sexually transmitted diseases clinic
}

\author{
G L RIDGWAY,* V MOSS,† G MUMTAZ,* W ATIA,† A M EMMERSON, † \\ AND J D ORIEL* \\ From the *Departments of Clinical Microbiology and Genitourinary Medicine, University College \\ Hospital; and the †Departments of Clinical Microbiology and Venereology, Royal Northern Hospital, \\ London
}

\section{Introduction}

Chlamydia trachomatis is now recognised as an important pathogen affecting the genital tract of men and women. ${ }^{12}$ Owing to cost and the need for a tissue culture laboratory, however, a routine culture service for this organism has not been widely available outside research centres. The arguments for and against the free availability of such a service have been discussed in detail..$^{3-6}$ All workers agree that, at the very least, a partial service for selected groups of patients should be more widely available.

Virus diagnostic laboratories tend to be localised on a regional basis, and it is in these departments that tissue culture expertise is readily available. We, therefore, examined the feasibility of a central reference laboratory sending prepared coverslip cell cultures to a peripheral diagnostic laboratory for inoculation, incubation, and interpretation.

\section{Patients and methods}

Unselected male patients attending a sexually transmitted diseases (STD) clinic (Royal Northern Hospital) with urethral discharge were included in the study. Patients receiving antimicrobial chemotherapy within the previous four weeks were

Address for reprints: Dr G L Ridgway, Department of Genitourinary Medicine, University College Hospital, Gower Street, London WCIE 6ẢU

Accepted for publication 8 February 1982 excluded. Specimens were taken from the dista urethra for Gram stain and culture for Neisserit gonorrhoeae. A cottonwool-tipped wire swab was़ inserted $4 \mathrm{~cm}$ into the urethra, rotated, and removed: The tip was cut off into chlamydia transporø medium $^{7}$ screened with gentamicin, vancomycin, an amphotericin B. Inoculated specimens were held as $4^{\circ} \mathrm{C}$ until transported to the local laboratory in the same hospital (usually within the working day). $O \AA$ arrival, one half of the transport medium wa $\vec{E}$ decanted and sent to the central laborator $\bar{y}$ (University College Hospital), usually within 29 hours. Both specimens were inoculated on to IUDR treated McCoy cells prepared at the centraps laboratory by the method described. ${ }^{7}$ Prepare coverslip monolayers were sent to the locap. laboratory from the central laboratory at twice weekly intervals. After centrifugation and incubation coverslip preparations were stained with Lugol's iodine and examined by light microscopy. Neither laboratory was at this stage aware of the other's findings. All slides were subsequently reviewed at the central laboratory and any discrepancies checked by a third observer. Statistical correlates were calculated using the $\chi^{2}$ test with Yates's correction.

\section{Results}

Specimens were obtained from the urethras of 2150 men. Seven of these specimens disrupted both laboratories' cell sheet. A further two specimens caused disruption of the local laboratory cell culture 
TABLE I Isolation of C trachomatis from urethral specimens with and without delayed inoculation

\begin{tabular}{|c|c|c|c|c|}
\hline \multicolumn{3}{|c|}{ Culture and interpretation by: } & \multirow[b]{2}{*}{ Total (\%) } & \multirow[b]{2}{*}{$\begin{array}{l}\% \text { Of confirmed } \\
\text { positives }\end{array}$} \\
\hline Local laboratory & $\begin{array}{l}\text { Central laboratory } \\
\text { (of same sample) }\end{array}$ & Central laboratory & & \\
\hline+ & + & + & $38(18 \cdot 45)$ & 69 \\
\hline+ & + & - & $12(5 \cdot 83)$ & $21 \cdot 8$ \\
\hline+ & - & - & $3(1 \cdot 46)$ & \\
\hline- & + & - & $2(0.97)$ & $3 \cdot 63$ \\
\hline - & - & + & $3(1 \cdot 46)$ & $5 \cdot 45$ \\
\hline- & - & - & $148(71 \cdot 84)$ & \\
\hline Total & & & 206 & \\
\hline
\end{tabular}

$+=$ Positive; $-=$ negative

only. The central laboratory culture for these two latter specimens gave a negative result for $C$ trachomatis. Thus, specimens from 206 men were available for comparison. Of these patients, 18 $(8 \cdot 7 \%)$ had gonococcal urethritis and 188 nongonococcal urethritis.

$C$ trachomatis was isolated from $58(28 \cdot 2 \%)$ of the specimens; in 38 the organism was isolated from cultures at both laboratories (table I). The local laboratory culture alone yielded $C$ trachomatis from 12 specimens and the central laboratory culture alone yielded $C$ trachomatis from three specimens. Three cultures reported as positive by the local laboratory were on review at the central laboratory thought not to grow $C$ trachomatis, and two cultures reported as negative by the local laboratory were positive on review.

Failure to isolate $C$ trachomatis at the central laboratory, although the local laboratory cultures gave positive results, was not related to the delay between collection of the specimen and the inoculation of the cell monolayer. There was, however, a strong association between the inclusion count in the local laboratory specimen and the isolation of $C$ trachomatis from both specimens (table II). This relationship was particularly significant when inclusion counts of $<25$ were compared with those of $>25(\mathrm{P}<0.001)$.

TABLE II Relationship of inclusion count to positivity in 48 specimens growing $C$ trachomatis

\begin{tabular}{lcc}
\hline & \multicolumn{2}{c}{ C trachomatis isolated from: } \\
\cline { 2 - 3 } Inclusion count & $\begin{array}{l}\text { First specimen } \\
\text { only }\end{array}$ & Both specimens \\
\hline$>300$ & 2 & 15 \\
$<300$ & 14 & 17 \\
Total & 48 & $(0 \cdot 05>\mathrm{P}<0 \cdot 02)$ \\
$>25$ & 5 & 27 \\
$<25$ & 11 & 5 \\
Total & 48 & $(\mathrm{P}<0 \cdot 001)$ \\
\hline
\end{tabular}

\section{Discussion}

C trachomatis is an important cause of genital infection in both men and women, and its accurate diagnosis in STD clinics is highly desirable. Often specimens are sent to a central laboratory for processing. Our results, however, show that over $20 \%$ of the $C$ trachomatis isolates would not have been detected if the specimens had been processed at the reference laboratory. Thus, apart from the convenience of culturing the specimen locally, the yield was considerably increased. The discrepancies between the two laboratories' interpretation of the same coverslip concerned low inclusion counts, which might have been subject to observer error under normal conditions. Of the three specimens giving positive results only in the central laboratory culture, two had low inclusion counts $(<10 /$ coverslip) and the remaining culture $>300$ inclusions/ coverslip.

The disruption of the cell sheet in nine cultures did not appear to be due to excess growth of $C$ trachomatis, as in two specimens the central laboratory culture result was negative without disruption of the cell sheet. This finding is in line with the toxin theory of Ngeow et al. ${ }^{8}$

The finding that the number of inclusion-forming units in the initial inoculum was related to the ability to isolate the organism after transport implied that the antigenic load was the limiting factor rather than delay in inoculating cell cultures. We have found this to be true of specimens from the eyes of babies with chlamydial ophthalmia (where the antigenic load is often very high), which are more likely to survive transport to a distant laboratory than specimens from the adult genital tract.

The bulk preparation of coverslip cultures is more economical of staff and materials than production by a number of smaller units. Culture preparation and the maintenance of cell lines are the most time consuming aspects of the isolation of $C$ trachomatis, since they require a high level of ability in tissue 
culture techniques. In contrast, the inoculation, incubation, and interpretation of cultures are within the capabilities of the trained staff of a routine diagnostic laboratory and do not require expensive equipment. The provision of a temperaturecontrolled centrifuge is desirable, but such a piece of apparatus will have other uses within the department. A proper technique of specimen collection is important or a low (and potentially misleading) isolation rate will be obtained.

Large-scale screening methods for $C$ trachomatis, such as described by Mallinson et al, ${ }^{9}$ do not overcome the problems of transport and the need to store specimens before inoculation. The provision of prepared cell cultures from a central laboratory makes the culture of $C$ trachomatis economic and convenient. Taking the cultures to the patient, as suggested by Ngeow et al, ${ }^{8}$ might provide a useful refinement to this technique.

We thank the staff of the Microbiology Departments and Sexually Transmitted Diseases Clinics at University College Hospital and the Royal Northern Hospital for their assistance and co-operation with this study.

\section{References}

1. Schachter J. Chlamydial infections. $N$ Engl J Med 1980 298: 428-35; 490-5, 540-9.

2. Taylor-Robinson D, Thomas BJ. The role of $C$ trachomatis in genital tract and associated infections. J Clin Pathol 1980; 33:205-33.

3. Richmond SJ, Oriel JD. Recognition and management of genital chlamydial infection. $B r$ Med $J 1978 ; \mathrm{ii}: 480-3$.

4. Willcox JR, Fisk PG, Barrow J, Barlow D. The need for a chlamydial culture service. Br J Vener Dis 1979;55:281-3.

5. Richmond SJ, Paul ID, Taylor PK. Value and feasibility of screening women attending STD clinics for cervical chlamydial infections. Br J Vener Dis 1980; 56:92-5.

6. Schachter J, Grossman M. Chlamydial infections. Ann Rev Med 1981;32:45-61.

7. Reeve P, Owen J, Oriel JD. Laboratory procedures for the isolation of $C$ trachomatis from the human genital tract. J Clin Pathol 1975; 28:910-4.

8. Ngeow YF, Munday PE, Evans RT, Taylor-Robinson D. Taking cell culture to the patient in an attempt to improve chlamydial isolation. Br J Vener Dis 1981; 57:44-6.

9. Mallinson H, Sikotra S, Arya OP. Cultural method for large scale screening for Chlamydia trachomatis genital infection. $J$ Clin Pathol 1981;34:712-8. 